\title{
A Systemic Functional Analysis of Two Multimodal Covers
}

\author{
María Martínez Lirola \\ University of Alicante \\ maria.lirola@ua.es
}

\begin{abstract}
Our society is influenced by new texts, which are clearly characterised by the increasing dominance of the visual mode; this implies that new literacies need to be developed as a way of enabling the readers to question the texts they are exposed to.

We argue for a multimodal and situated approach for understanding and interpreting writing on magazine covers because they are examples of an increased emphasis on modes of representation other than the written, especially an increased dominance of the visual mode. This is the reason why we are going to analyse two covers of free British magazines (published in London on July 14,2003 ) to see the different resources they use to attract people's attention and to encourage readership, particularly because they were delivered at the exit of underground stations.

Systemic Functional Linguistics will be our theoretical framework in this article because for this linguistic school the text is the basic unit of analysis and it studies language in relation to society. SFL will help us to understand why a text is written as it is by paying attention to its context and textual organisation.
\end{abstract}

\section{Introduction}

It is evident that we need to be active participants in today's society, which is why we need to develop a critical perspective to read texts that employ a variety of modes to convey meaning. Our society is influenced by the presence of new texts, which are clearly characterised by the increasing dominance of the visual mode. This implies that new 
literacies, such as critical media literacy or critical literacy, need to be developed as a way of enabling the readers to develop a critical attitude to the texts they are exposed to, in Kress' words (2003: 61), we need to analyse "how the modes of image and writing appear together, how they are designed to appear together and how they are to be read together".

The discourse of covers is multimodal in nature and presents an increased emphasis on modes of representation which are not written texts, especially an increased dominance of the visual mode to catch people's attention. This leads us to consider how the visual elements and contexts of a text contribute to our overall experience of the text because there is a clear combination of verbal and visual meanings. The context in which these texts are created and the one in which they are going to be used is crucial, as the following quotation corroborates: "Contexts are not simply containers within which actions, practices, and activities occur. Instead, they are dynamic streams of overlapping and integrated discourses, spaces, sociocultural practices, and power relations". (Kambeleris and de la Luna, 2004: 243)

When we read any page we have certain expectations, and the page should be visually designed to meet these expectations. Since the texts under analysis are the covers of different magazines and they need to attract the reader's attention, it is expected that they connote their visual elements by using many colours and images, as we will see in the analysis in section three of this article. As Frances (2004: 124) states: "That we associate particular visual arrangements with different genres of writing means that the visual arrangements do some of the work of the genre. This means, then, that the visual arrangements can be analysed in terms of the genre work they do".

We argue for a multimodal and situated approach for understanding and interpreting writing on covers. This is why we are going to analyse two covers of free British magazines (published in London on July 14, 2003; see Appendix) to see the different resources they use to attract people's attention and to persuade them to encourage readership, particularly because they were delivered at the exit of underground stations. We will analyse the two covers as multimodal texts, taking into consideration that the text can be a complex phenomenon (Halliday and Matthiessen, 2004: 3); we will also consider that " [...] the interpretation of texts is structured not only by 'what the text says', but also by contextually specific rules of interpretation, [...]" (van Leeuwen, 2005: 83)

Since the text will be the basic unit of our analysis Systemic Functional Linguistics (hereafter SFL) will be used as an analytical framework, because this linguistic school studies language in relation to society and analyses the main reasons for choosing between some linguistic forms or others, which is always determined for the function that those linguistic forms have in society. We are interested in a systemic functional approach to language because of the interrelationship between language, text and the contexts in which those texts occur, and because it includes a social perspective in the study of language.

SFL concentrates on the analysis of authentic products of social interaction (texts), considered in the social and cultural context in which they take place. The most generalized application of systemic linguistics is "to understand the quality of texts: why a text means what it does, and why it is valued as it is". (Halliday, 1994: xxix). 
Systemics describes a text in terms of the different choices of language we find in a given text and in the way a text function's realises what is happening (ideational metafunction); how it interacts with the reader or hearer (interpersonal metafunction), and how a text coheres (textual metafunction). As Martin (1992: 493) proposes: "texts are social processes and need to be analysed as manifestations of the culture they in large measure construct". Systemic linguists place considerable emphasis on the idea of choice, i.e., we view language as a network of interrelated options from which speakers and writers can select according to their communicative needs.

Having SFL as a framework helps us understand why a written text is used in the way it is. It does so by paying attention to its context and textual organization because it enables an indepth study of the construction of meaning in the text. The analysis will reveal that the visual elements and the context of a text influence our overall experience of the text, because texts have to be understood in their context since they represent the reality that surrounds them as Kambeleris and de la Luna (2004: 241) discuss:

Texts are not simply denotative devices that stand for and correspond to "real-world" referents that lend them meaning. Instead, they give shape to the reality they implicate as much as they present or represent it. Because texts are indexical-pointing to the contexts in which they have concrete meanings and functions-paying careful attention to the formal (semiotic) properties of texts can tell us a lot not only about the internal organization of the texts themselves but also about their authors, contexts of use, audiences, and so on.

As readers, we have to be able to interpret critically the texts we interact with on a daily basis and become more analytical in our literacy practices around texts. In this way we will develop a critical literacy. That is why we need to use different tools to analyse in detail, and to talk about a text in any mode - to develop skills about texts and to improve our understanding of multimodal texts. In this sense, Kress and van Leeuwen's (1996) Reading Images: A Grammar of Visual Design can be very useful since it is a grammar for the visual mode based on the principles of SFL.

The logical starting point for analysis of written texts is to consider the meaning of the text since all texts are about something, as Bazerman and Prior (2004: 2) declare: "To understand writing, we need to explore the practices that people engage in to produce texts as well as the ways that writing practices gain their meanings and functions as dynamic elements of specific cultural settings".

We are going to analyse the different resources used in the covers under analysis to convey messages and to catch the readers' attention. With the analysis, we intend to answer the following questions: "Who and what are the kinds of people, places and things depicted in this image, and how do we recognize them as such? and What ideas and values do we associate with these depicted people, places and things, and what is it that allows them to do so?" (van Leeuwen, 2001: 92) 


\section{Analysis of visual grammar's concepts and linguistic features of the written text in two multimodal covers}

The texts on the two magazines are multimodal because they include two different modes: visual and written. In this section we intend to highlight the different values of these texts. We do not understand the multimodal text as one that can be divided into different semiotic channels On the contrary, the multimodal text is a unity in which we can observe different resources (Thibault, 2000: 321).

Following Kress and van Leeuwen (1996) we can point out that the main visual features of magazine texts are: colour, layout, salience, framing and photographs. It is evident that colour is of great significance since it is used to attract the reader's attention. As Kress and van Leeuwen (2002: 347) declare, the colours of the text, including clothing, are used to denote specific aspects of the person or character. In the backgrounds, we normally find bright colours, which establish a contrast with the colours used for the writing. Normally the background's colour makes it easier to see and read the written message. The colour always suggests something- some colours make the reader comfortable and others can make him/her feel uncomfortable. The selection of colours normally has an impact on the feelings of the reader, drawing an emotional response to the text (Kress and van Leeuwen, 2002: 348).

The colour of backgrounds creates a specific context. In this way we can speak about cohesion in the use of colours. In this kind of text, the written text becomes part of the visual and usually contrasts with the background; it is normally black or write or links with another element on the page, for example the colour of the heading. The dominance of colour background or image are designed to attract the reader's attention, which normally implies the reader's desire to engage with the text i.e., people are normally attracted by the colours, which is why we can state that colours encourage interaction.

Colour is a very important aspect in this kind of magazines because people received these two different magazines on the same day. Therefore, the fact that they decide to look at one of them or read some of the articles inside is favoured by the colours they use and the personality of the reader who has to ask himself/herself: 'Since I do not have much time, which magazine shall I read until I get to the underground station where I get off?' . Colours and photographs have an impact on the readers and have a lot to do with the decision they make.

In the two covers we find a clear differentiation of colours- brightness is important in these texts, the written text is easy to read and there is a clear division of the information according to its value: the left side of the page is associated with the Given and the right side of the page is associated with the New, i.e., with the most important part of the information. In visual discourse, right pages are normally dominated by large and salient photographs; on the other hand, left pages contain mostly verbal text. As Kress and van Leeuwen declare (1996: 187), the information value we find on the left and on the right is Given and New respectively: 
[the Given is defined as] something the viewer already knows, as a familiar and agreed-upon point of departure for the message. For something to be New means that it is presented as something which is not yet known, or perhaps not yet agreed upon by the viewer, hence as something to which the viewer must pay special attention.

The way the page is arranged is intended to guide the reader's attention to certain parts of the text: the image/s, the written text, the heading, etc. What is found at the top of the page is given prominent position, especially if it is in the right-hand side corner. Images normally stand out since they are what we see first. Normally, half the page is taken up by the image/s and the other half is taken up by the written text, as we can clearly see in the covers of Gat and Ms London (figures 1 and 2; see Appendix).

Headings are usually large and bold, and placed at the top of the page. Images and written text blocks may be placed on the right-hand or left-hand side of the page or on the top or bottom. The left-hand side, the space for Given information, places elements of less importance than those placed on the right side because they are assumed to be known by the reader; the right-hand side, the space for the New information is where the most important elements are located and where the reader should concentrate his/her attention (Kress and van Leeuwen, 1996: 186).

Salience can create a "hierarchy of importance among the elements, selecting some as more important, more worthy of attention than others" (Kress and van Leeuwen, 1996: 212). Salience is realised through size, colour, colour contrasts, tonal contrast and placement of the page, thus drawing the reader's gaze to an element of the text. Size is normally a major factor of salience. In combination with colour, the heading is usually large and stands out. In magazines the image is normally the most salient on the page because it takes up a large part of the page and seems to dominate the written text. This contrasts with the written text blocks, which are normally in small font and are rarely the most salient feature.

Frames are another important visual feature. They are normally used to highlight the written text or an image; the frame has an effect on our perception of elements as separate units of information. Kress and van Leeuwen (1996: 183) explain that "the presence or absence of framing devices (dividing or framing lines) which connect or disconnect elements, signifying that they belong or do not belong together".

Photographs are often used to bring a sense of immediacy and reality to the text in a way that promotes interaction with the reader, as we will see in the following paragraphs. Covers, as any text, are semantic units and consist of the ideational, interpersonal and textual meanings which form a coherent whole. In the following section we will comment on different aspects related to the three functions of language ${ }^{1}$ : all aspects related to cohesion have to do with the textual function of language; we concentrate on the ideational function because we see the covers as a whole and then we observe the different parts in which we can divide them; the analysis of colours, of appraised vocabulary to evaluate the world, which is related to the interpersonal function.

The visual elements of covers (colours, shapes, written texts and images) are carefully 
chosen because they perform a persuasive task. For example, when choosing colours, the designer normally chooses a combination of bright and dark since this mixture attracts the reader's attention.

The visual aspects of texts are to be understood as embedded in the social context where they are used because the designer is influenced by the social circumstances in which the text is composed, and because he/she also considers the circumstances in which these texts will appear and do their job. The designer creates the page according to what he wants the reader to see first on a page and the mood that the text should create. $\mathrm{He} / \mathrm{sh}$ creates a relationship between the different parts of the text that contribute to the internal coherence and cohesion. This internal coherence is related to the logic of the text. In Halliday's words (1994: 339):

For a text to be coherent, it must be cohesive; but it must be more besides. It must deploy the resources of cohesion in ways that are motivated by the register of which it is an instance; it must be semantically appropriate, with lexicogrammatical realizations to match (i.e. it must make sense); and it must have structure.

The two covers try to persuade readers that any information they take from these pages is as close to the present moment as possible. Readers see first what is on top of the page (because we have been taught to read starting at the top) and then they move down. The different ways in which the elements of a multimodal text are placed have an effect on how our attention moves over the page. The size and colour of something and its placement at top or bottom, left or right, has an influence in the way we perceive the page since there is normally a hierarchical relationship between elements.

Since these magazines are delivered to readers who are getting the underground in the early morning, which implies that most of them are in a hurry to get to work or somewhere else, the persuasive effect of the cover's colour is crucial: it must invite a busy reader to open the magazine and read. Since he/she receives different magazines at the same time, the cover has an important role.

It is evident that, among the two covers, the elements given the most visual attention are the headings, which stand out because of their placement at the top, and the images because of their size. In the two covers analysed, we can observe that there is one significant image which takes up the largest part of the page. Apart from the image we find written information in the left side of the page; the written text can be considered a clarification of the image. Roland Barthes (1977: 40) coined the term 'anchorage' for cases like these in which the text helps to understand the image and vice versa.

The letters chosen to present the written texts in the covers are big enough to be read easily. There is enough space between the words and the different written blocks. As expected, the heading with the name of the magazine is placed at the top of the page in capital letters: they are white in Gat, which contrast with the black top the woman is wearing, and red in Ms London because this is the colour of the woman's dress. Although this is not the norm, the heading of the two covers under analysis is short so that it is easy 
to remember: Gat and Ms. London. Gat is surrounded by a blue square, the same colour as some of the written blocks, giving cohesion to the cover.

In Ms London, the heading is in red, which matches the colour of the woman's dress and some of the written blocks; the red writing stands out against the white background colour. This colour makes the red of the dress, the title, and the written blocks stand out even more. The letter blocks are placed on the left, on the Given part. Some of them are red and others are black, two colours that match well and establish a clear contrast. Red matches with the woman's dress and the title of the magazine and at the same time there is a contrast with the black of her shoes, hand bag and bracelet.

Since red is a bright colour, a symbol of passion in our culture, there are two lines bigger than the rest which are written in pink. This clearly draws the reader's attention to them and refer to the most important article we can find in the magazine since they refer to 'Gwyneth Paltrow's BIG MAKE OR BREAK', where we can perceive an antithesis. It is a fact that pink is associated with girls. It is soft, pretty, a stereotypical feminine colour which links to the sweet and sensual image of the woman presented in the picture.In this cover we also find a written block on the right, in the New part as a way of showing that this is an important part of the information. The right side of the written text block aligns with the shape of the woman's body.

As regards the written text, it is important to highlight that in the cover we find several questions that invite the reader to read the magazine and find the answers: 'How Hollywood divas handle the put down', 'Got the ring, got the bloke but will she and Chris Martin falter at the altar?'Also interesting is the use of appraisal at the beginning of the cover: dawn, bitches and the put down. In this way the reader's attention is attracted because those words have negative connotations that cause shock because that is not what we would expect on a cover.

The woman in Ms London is moving, which suits the intended audience: people who are starting their day going somewhere by underground. Her mouth is open as if she is going to say something. The background between the written blocks and the picture frames the sections, making them distinct from each other, while at the same time associated by the alignments chosen.

In the magazine Gat, we find the written text on the left, the space of the Given, and the photograph of a blond, good looking woman is placed on the right, the place of the New. The heading is on a blue square and there is a sub-heading 'Girl about town' in black small capitals. The background of this page is quite dark-dark blue, almost black- but since the letters are white and bright blue, the written text blocks can be read easily.

The exclamation on the word yo! and the imperative 'bring back the mingers' are used for emphasis and to create interaction with the reader. Apart from this, it is also important to note the use of some contractions and short forms (fests, we're, telly), which give an informal quality to the cover in such a way that it can connect with the reader. In the same way, the use of appraisal -'Nasty niffs', bitch, evil, sex- catches the reader's attention.

As we have already commented with Ms London, the background between the written blocks and the picture frames the sections, distinguishing from each other. On the other 
hand, the picture is different from the one in the previous magazine because the woman in this picture seems to be static, observing, with her mouth closed. Her hair style covers part of her eyes, which makes her enigmatic. Her dark blue necklace and the small part of the top of her black dress clearly match the dark blue of the background of the page. The makeup on her lips and around her eyes is the same colour, i.e., colour creates cohesion.

Next to the heading, on the right side at the very top of the page, we find two lines of written text in white and blue, the two colours combined in the writing of the entire cover: 'summer fests postglastonbury - best of the rest'. Since they are placed at the top of the page in the side where new information is placed, they are given prominence.

\section{Comparison of both covers}

In the following paragraphs, we are going to establish a parallelism between the cover of Gat and Ms London in several aspects:

Firstly, there is an overlap between both images and a part of the written block on the left, at the bottom of the page, which creates a cohesive link with the image through association by placement. In both covers, the written text block and the images are placed on the same background, creating an associative link between the two elements, i.e. the writing and the image go together. The background contrasts strongly with the two photographs: with the red dress in Ms London and with the white skin and the blond hair in Gat.

Another interesting parallelism is that both women are blond, although one of them has short hair and the other has long hair. The woman in Ms London is dressed in red and the woman in Gat is dressed in black, two colours that clearly represent the trend in woman's fashion, and they try to get an emotional response from the reader. Both women are serious and don't look very natural i.e., they can be considered ideal women because they would need hair stylist, make-up artists, etc. to get that look.

It is evident that, in both covers, the image of the woman and the headings are the most salient elements on the page. In both cases, the image is on the right side, the prominent place for information, and the written text is on the left because it is secondary to the image. Thus it can be deduced that if the image does not catch the reader's attention it is very likely that he/she will not read anything written on the cover or inside the magazine. In this way the image takes up most of the right side of the article and dominates the written text. The woman's face appears on the upper part of the page and in Ms London, it covers a great part of the ND of the word London, which is the main one we find in the heading.

In Ms London the placement of the writing on a pale background (white and blue) facilitates reading, not just the viewing of the page or the image alone. Written texts in different sizes are more likely to be read than the rest of the written text. It is interesting to note that the date of the magazines (14 ${ }^{\text {th }}$ July 2003) is placed in two different areas of the covers: Ms London places it at the very top of the page, on the right side, on the heading and it is in black and Gat uses the white colour and places it on the left, after the heading 
and the sub-heading. The different choices (we cannot forget that SFL proposes a grammar of the language as choice and observes how the choices at all strata build the context), i.e. size, colour, brightness versus images have an effect on the meanings made.

Multimodal resources can construct stereotypes, as happens in Ms London and Gat where we are presented two pictures of young beautiful women, representing the type of woman most people want to become in European countries. As Cameron (1997: 49) proposes: "Gender has constantly to be reaffirmed and publicly displayed by repeatedly performing particular acts in accordance with the cultural norms (themselves historically and socially constructed, and consequently variable) which define 'masculinity' and "feminity".

The red colour, for example, is symbol of passion in our culture and since it is a bright colour, it is also associated with abundance, i.e., there are cultural values associated to colours and images. From what we have said in the previous lines, it is evident that there is a link between the purpose of the text, its structure, and its major language and visual features because it is for a very explicit audience: people getting the underground in the early morning: professionals, semi-professionals, commuters, etc. As Hyland (2000: 10) acknowledges: "Readers must be drawn in, influenced and persuaded by a text that sees the world in similar ways to them, employing accepted resources for the purpose of sharing meanings in that context".

We can see how these covers contain cultural attitudes and ideologies: in both we find two images of women in the New part, i.e., they are the most important part of the text; they are very attractive and good looking. We can see an overall balance and harmony in these covers. At the same time, how these covers are read depends on the reader's ideology, although in our society images are very important, and being good looking and beautiful seems to be essential. That is why we agree with Beard (2001: 4) when he considers that: "A text cannot have an existence independent of its readers, who recreate the text through bringing their own culturally-conditioned views and attitudes to bear on it".

\section{Conclusions}

In the two texts under analysis, the designer has chosen a wide range of language features and visual devices to set up the structure of the text, avoiding repetitiveness. This analysis has made us reflect on the purpose, the appropriate structure and the common features of this type of text. In this article we have analysed some aspects of the style, structure, visual and linguistic features of the multimodal texts found in two free English magazines.

Texts are always produced in the socio-cultural context of their time, and we gain a better understanding of them by taking context into account. Taking into consideration the contextual issues surrounding texts involves a kind of social relationship between writer and reader. Readers need to see social interactions (writer and reader) through the process of reading multimodal texts, keeping in mind that texts are always interpreted according 
to the cultural frame of the individual.

The covers analysed are effective texts that contain ideas consistent with the cultural conventions in which they are written, i.e., there is a connection between form and meaning. The two covers are designed to be read as well as seen by the readers. It is evident that a text cannot exist independent of its readers- they always bring their culture and ideology to the text, recreating and enriching it. In this way, the readers become active and not just passive recipients- they add meaning to the text. As critical readers, we have to be aware of the social purpose of these multimodal texts. Texts are always produced inside their context. It is important that we keep in mind that we as readers can understand a culture by paying attention to all texts that are produced in that culture.

Having SFL as a framework, we have concentrated on the visual mode, paying attention to aspects such as the use of colour, the photographs or pictures and the writing and the background. It is evident that in the two covers there is an image of significance and the written text can be considered a visual element with different size, colours and the use of bold for emphasis.

In this analysis we have asked the following questions to concentrate on the main aspects of the multimodal analysis we have followed: 'What is the first thing you see when you look at the page?', 'Where is the image placed on the page?', 'What colours are used and how do they match?', 'Where is the written text placed and how is it in comparison to the image?' and above all, 'How does a multimodal text communicate, how does it establish relationships between the represented world and the reader?'

We have discussed the placement of the image and writing in relation to the reasons a designer has to place an image on the right or left, considering that the right side is the space for the New, i.e., the area where the designer wants the reader to look or read first. We have also given attention to the types of images used, which is really significant in Ms London and Gat, where we find the typical, fashionable, good looking, slim woman.

This analysis is not an end in itself because our purpose is to make people reflect on the persuasive effect the reading of a multimodal text has on the reader. Our intention is to highlight that the construction of multimodal texts is not random because the designers choose images carefully and place them in a very precise way. The colours are also chosen carefully, normally bright and dark together, because the colour is very important so that it suits the image, the size and type of writing.

The learning of visual grammar has a definite impact on readers' understanding and knowledge of multimodal texts in magazines, in other words, visual grammar makes readers become more critical and be able to decode the hidden messages on multimodal texts. Multimodal awareness provides multiple perspectives on how relations between discourses and society work and facilitate a better understanding of the way discourses work in different societies.

Multimodal texts hide interactive strategies from the readers. The designer chooses every aspect taking part in this kind of texts carefully. Choices of how to organise texts are influenced by context and the imagined audience, i.e., the text acquires meaning in the context in which it is produced, distributed and consumed. 
Notes

1. The three metafunctions of language used in SFL, namely ideational, interpersonal and textual, are referred to as representational, interactive and compositional by Kress and van Leeuwen (1996) but in this paper we will use the systemic terminology.

\section{References}

Barthes, Roland (1977): "Rhetoric of the Image". In R. Barthes (S. Heath, ed. and trans.) ImageMusic-Text. London: Fontana, 32-51.

Bazerman, Charles and Paul Prior (eds.) (2004): What Writing Does and How it Does it. An Introduction to Analyzing Texts and Textual Practices. Mahwah, New Jersey: Lawrence Erlbaum Associates.

Beard, Adrian (2001): Texts and Contexts. Introducing Literature and Language Study. London/New York: Routledge.

Cameron, Deborah (1997): "Performing gender identity: young men's talk and the construction of heterosexual masculinity". In S. Johnson and U. H. Meinhof, eds., Language and Masculinity. Oxford/Cambridge: Blackwell Publishers, 47-64 .

Frances, Anne (2004): "The multiple media of texts: How onscreen and paper texts incorporate words, images and other media". In C. Bazerman and P. Prior, eds., What Writing Does and How it Does it. An Introduction to Analyzing Texts and Textual Practices. Mahwah, New Jersey: Lawrence Erlbaum Associates, 123-163.

Halliday, Michael Alexander Kirkwood (1994): An Introduction to Functional Grammar. Second edition. London: Arnold.

Halliday, Michael Alexander Kirkwood and Christian M.I.M Matthiessen (2004): An Introduction to Functional Grammar. Third edition. London: Hodder Arnold.

Hyland, Ken (2000): Disciplinary discourses: Social interactions in academic writing. Essex: Pearson Education.

Kambeleris, George and Lenora de la Luna (2004): "Children's Writing: How Textual Forms, Contextual Forces, and Textual Politics Co-Emerge". In C. Bazerman and P. Prior, eds., What Writing Does and How it Does it. An Introduction to Analyzing Texts and Textual Practices. Mahwah, New Jersey: Lawrence Erlbaum Associates, 239-277.

Kress, Gunther (2003): Literacy in the New Media Age. London: Routledge.

Kress, Gunther and Teun van Leeuwen (1996): Reading Images: The Grammar of Visual Design. London: Routledge Press.

. (2002): "Colour as a semiotic mode: Notes for a grammar of colour". Visual Communication, 1 (3): 343-368.

Martin, James. R. (1992): English Text: System and Structure. Amsterdam/ Philadelphia: John Benjamins.

Thibault, Paul (2000): “The multimodal transcription of television advertisement: theory and practice". In A. Baldry, ed., Multimodality and Multimediality in the Distance Leaming Age. Campobasso:Palladino Editore, 311-385.

Van Leeuwen, Teun (2001): "Semiotics and Iconography". In T. Van Leeuwen and C. Jewitt, eds., The Handbook of Visual Analysis. London: Sage, 92-118.

(2005): Introducing Social Semiotics. New York: Routledge. 
Appendix
